\title{
Circular Cosmic String Loop in Brans-Dicke Theory
}

\author{
A. Barros ${ }^{\dagger}$, A. A. Sen ${ }^{\ddagger *}$, and C. Romero ${ }^{\star}$ \\ ${ }^{\dagger}$ Departamento de Física, Universidade Federal de Roraima \\ 69310-270 Boa Vista, RR, Brazil \\ ${ }^{\ddagger}$ Harish-Chandra Research Institute \\ Chhatnag Road, Jhusi, Allahabad, 211019, India \\ * Departamento de Física, Universidade Federal da Paraíba \\ Caixa Postal 5008, 58059-970 João Pessoa, PB, Brazil
}

Received on 15 March, 2001

\begin{abstract}
The gravitational field of a stationary circular cosmic string loop, externally supported against collapse, is investigated in the context of Brans-Dicke theory in the weak field approximation of the field equations. The solution is quasi-conformally related to the corresponding solution in Einstein's General Relativity(GR) and goes over to the corresponding solution in GR when the Brans-Dicke parameter $\omega$ becomes infinitely large.
\end{abstract}

\section{Introduction}

Phase transition in the early universe might have produced some topological defects [1]. Amongst these defects, cosmic strings, have attracted a lot of interest for various reasons [2]. For instance, they are capable of producing observational effects such as double images of quasars and are considered to be possible candidates as seeds for galaxies formation.

In some gauge models strings do not have any end, and thus are either of infinite extent form or closed circular loops. One of the most notable features of the gravitational field of a straight infinite cosmic string is the presence of an "angular deficit", in an otherwise Minkowskian spacetime, having a magnitude related to the linear energy density $\mu$ of the string by the equation $\delta \Phi=8 \pi G \mu$. In fact, this angular deficit plays a key role for the production of double images of quasars [3].

The deficit angle model is widely believed to be a good approximation for describing a spacetime exterior to the string core. Frolov, Israel and Unruh (FIU) [4] used this approximation for studying a closed circular cosmic string loop at a moment of time symmetry. With the help of the initial value formulation [5], they produced a family of momentarily stationary circular cosmic strings, which are regarded as thin loops either at the time of formation or at the turning point of expansion and collapse. An important assumption in this work is that all points on the circular string be conical singularities with angular deficit equal to that of a infinite straight string of equal linear energy density. Hughes, McManus, and Vandyck(HMV) [6] investigated further the problem of angular deficit of a circular string. They considered a weak field stationary solutions of Einstein's field equations for a thin circular string and also established that radial stress should be introduced to support the string loop against possible gravitational collapse. They determined the form of the radial stress from the stress energy conservation relations. The main result of their study is that in weak field approximation a circular string produces, locally, along the string the same angular deficit as does a straight infinitely long string with the same linear energy density. In another work, McManus and Vandyck [7] considered a string loop with a rotation of the loop which provides the necessary centrifugal reaction partially or fully in order to avoid the possible gravitational collapse.

It turns out that at sufficient high energy scales it seems likely that gravity is not given by the Einstein's action, but becomes modified by the superstring terms. In the low energy limit of this string theory one recovers Einstein's gravity along with a scalar dilaton field which is non-minimally coupled to gravity [8]. On the other hand, scalar tensor theories, such as Brans-Dicke theory(BD) [9], have been considerably revived in recent years. It was shown by La and Steinhardt [10] that because of the interaction of the BD scalar field with the Higgs type sector, the exponential inflation in Guth's

*e-mail:anjan@mri.ernet.in 
model [11] could be slowed down to power law one and the graceful exit in the inflation is thus completed via bubble nucleation. Although dilaton gravity and BD theory arise from entirely different motivations, it can be shown that the former is a special case of the latter, at least formally [12]. Another motivation for studying gravitational properties of defects in BD theory is that the only defects we can hope to observe now are those formed after or near the end of inflation, and the formation of such superheavy defects is relatively easy to arrange in a Brans-Dicke type theory [13].

In the present work we have studied the gravitational field of a stationary circular cosmic string loop in Brans-Dicke theory in the weak field approximation of the field equations.

The field equations in the BD theory are written in the form

$$
\begin{gathered}
G_{\mu \nu}=8 \pi \frac{T_{\mu \nu}}{\phi}+\frac{\omega}{\phi^{2}}\left(\phi_{, \mu} \phi_{, \nu}-\frac{1}{2} g_{\mu \nu} \phi_{, \alpha} \phi^{, \alpha}\right) \\
+\frac{1}{\phi}\left(\phi_{, \mu ; \nu}-g_{\mu \nu} \square \phi\right), \\
\square \phi=\frac{8 \pi T}{2 \omega+3},
\end{gathered}
$$

where $\phi$ is the scalar field, $\omega$ is the BD parameter and $T$ denotes the trace of the energy-momentum tensor $T_{\nu}^{\mu}$ [9]. In the weak field approximation of BD theory one can assume $g_{\mu \nu}=\eta_{\mu \nu}+h_{\mu \nu}$ where $\left|h_{\mu \nu}\right|<<1$ and $\phi(r)=\phi_{0}+\epsilon(r)$ with $\left|\epsilon / \phi_{0}\right|<<1$ where $1 / \phi_{0}=G_{0}=$ $\frac{(2 \omega+3)}{(2 \omega+4)} G$ in order to have a Newtonian limit for the BD theory [9].

It has been shown recently by Barros and Romero [14], that in the weak field approximation the solutions of the BD equations are related to the solutions of linearized equations in GR with the same $T_{\nu}^{\mu}$ in the following way: if $g_{\mu \nu}^{g r}(G, x)$ is a known solution of the Einstein's equations in the weak field approximation for a given $T_{\nu}^{\mu}$, then the BD solution corresponding to the same $T_{\nu}^{\mu}$, in the weak field approximation, is given by

$$
g_{\mu \nu}^{b d}(x)=\left[1-G_{0} \epsilon(x)\right] g_{\mu \nu}^{g r}\left(G_{0}, x\right)
$$

where $\epsilon(x)$ must satisfy

$$
\square \epsilon(x)=\frac{8 \pi T}{(2 \omega+3)},
$$

and $G$ is replaced by $G_{0}$ defined previously. Hence, to get the spacetime for circular string loop one has to solve the equation (1.4) with appropriate $T_{\nu}^{\mu}$ for the circular cosmic string loop.

We have taken the form of the energy momentum tensor for the string loop as proposed by Hughes et al. $[6]$ :

$$
\begin{gathered}
T_{t}^{t}=-\mu \delta(r-a) \delta(z) \\
T_{\phi}^{\phi}=k \delta(r-a) \delta(z)
\end{gathered}
$$

$$
T_{r}^{r}=\frac{k}{r} \Theta(r-a) \delta(z)
$$

in which $\Theta$ denotes the Heaviside step function. Here we are considering an infinitely thin loop of string with radius $a$, lying in the $x-y$ plane and centered at the origin. For a circular string $T_{\phi}^{\phi}$ plays the same role as that of the longitudinal stress $T_{z}^{z}$ for a straight string and $T_{r}^{r}$ is the external radial stress required for supporting the loop against collapse and hence it is not localized on the string.

With (1.5), equation (1.4) becomes

$$
\begin{gathered}
\nabla^{2} \epsilon=\frac{8 \pi}{2 \omega+3}[(k-\mu) \delta(r-a) \delta(z) \\
\left.+\frac{k}{r} \Theta(r-a) \delta(z)\right]
\end{gathered}
$$

Writing $\epsilon=\epsilon_{1}+\epsilon_{2}$, we can separate equation (1.6) into

$$
\begin{array}{r}
\nabla^{2} \epsilon_{1}=\frac{8 \pi}{2 \omega+3}[(k-\mu) \delta(r-a) \delta(z)] \\
\nabla^{2} \epsilon_{2}=\frac{8 \pi}{2 \omega+3}\left[\frac{k}{r} \Theta(r-a) \delta(z)\right]
\end{array}
$$

The solutions of the equations above can be found easily by using toroidal coordinates $(\phi, \sigma, \psi)$ which are related to cylindrical coordinates $(\phi, r, z)$ by $[4]$

$$
\begin{gathered}
z=a N^{-2} \sin \psi \\
r=a N^{-2} \sinh \sigma \quad(0 \leq \sigma \leq \infty,|\psi| \leq \pi)
\end{gathered}
$$

where $N^{2} \equiv N^{2}(\sigma, \psi) \equiv \cosh \sigma-\cos \psi$. The surfaces $\sigma=\sigma_{0} \equiv$ constant are tori whose generating circles have radii $a \operatorname{csch} \sigma_{0}$ and $a \operatorname{coth} \sigma_{0}$. The $\operatorname{ring} r=a$, $z=0$ is now given by $\sigma=\infty$.

In toroidal coordinates the solution of equation (1.7a) now becomes[15]

$$
\epsilon_{1}=-2^{\frac{5}{2}} \frac{(k-\mu)}{(2 \omega+3)} N(\sigma, \psi) \frac{F(\tanh (\sigma / 2))}{\cosh (\sigma / 2)}
$$

where $F$ denotes complete elliptic integral of the first kind and the solution for $\epsilon_{2}$ becomes[6]

$$
\begin{aligned}
\epsilon_{2}= & -\frac{4 k}{(2 \omega+3)} N(\sigma, \psi) \sum_{n=0}^{\infty}\left[H_{n}(\sigma) P_{n-1 / 2}(\cosh \sigma)\right. \\
& \left.+G_{n}(\sigma) Q_{n-1 / 2}(\cosh \sigma)\right] \varepsilon_{n} \cos (n \psi)
\end{aligned}
$$

where

$$
\begin{aligned}
& H_{n}(\sigma) \equiv \int_{0}^{\sigma} d x N^{-1}(x, 0) Q_{n-1 / 2}(\cosh x) \\
& G_{n}(\sigma) \equiv \int_{0}^{\sigma} d x N^{-1}(x, 0) P_{n-1 / 2}(\cosh x)
\end{aligned}
$$

and $P_{n-1 / 2}^{m}$ and $Q_{n-1 / 2}^{m}$ are toroidal Legendre functions, $\varepsilon_{n} \equiv 2-\delta_{n}^{0}$, and also we have used the notation $P_{n-1 / 2} \equiv P_{n-1 / 2}^{0}$ and $Q_{n-1 / 2} \equiv Q_{n-1 / 2}^{0}$. Thus, 
from equation (1.3) the spacetime for a stationary circular cosmic string loop in BD theory in the weak field approximation is given by

$$
d s^{2}=\left[1-G_{0}\left(\epsilon_{1}+\epsilon_{2}\right)\right] d s_{H M V}^{2}\left(G_{0}\right)
$$

where $\epsilon_{1}$ and $\epsilon_{2}$ are given by equations (1.9a) and (1.9b), and $d s_{H M V}^{2}\left(G_{0}\right)$ is the metric obtained by Hughes et al[6] for circular string loop in GR with $G$ replaced by $G_{0}$.

To investigate the presence of conical singularities on the ring $\sigma=\infty$, we proceed as follows: first of all, for the scalar field solution in equation (1.9) we may add an arbitrary constant $A$ such that

$$
\epsilon=\epsilon_{1}+\epsilon_{2}+A
$$

For $\sigma \rightarrow \infty$ one can find the asymptotic behaviour of $\epsilon_{1}$ and $\epsilon_{2}$ from equations (1.9) which is given by[6]

$$
\begin{gathered}
\epsilon_{1} \rightarrow-\frac{4(k-\mu) \sigma}{(2 \omega+3)} \\
\epsilon_{2} \rightarrow 0
\end{gathered}
$$

Hence, we have

$$
1-G_{0} \epsilon=1+\frac{4 G_{0}(k-\mu) \sigma}{(2 \omega+3)}-G_{0} A
$$

Then, for the surface $t=$ constant and $\phi=$ constant and using the toroidal coordinates one can obtain for the metric (2.1)[6]

$$
\begin{gathered}
d s^{2}=\left[1+\frac{4 G_{0}(k-\mu) \sigma}{(2 \omega+3)}-G_{0} A\right] \\
{\left[4 a^{2} e^{-2 \sigma} e^{4 G_{0}(\mu-k) \sigma+2 b}\left(d \sigma^{2}+d \psi^{2}\right)\right]}
\end{gathered}
$$

where $b$ is the dimensionless combination of all additive constants appearing in the solutions. Now, let us make the following coordinate transformation:

$$
\begin{gathered}
\psi=\theta-\pi \\
2 a e^{-\left(\sigma+2 G_{0}(k-\mu) \sigma-b\right)}=r
\end{gathered}
$$

Given that $b$ is a first order term in this approximation [6] we can write

$$
\begin{aligned}
& 1+\frac{4 G_{0}(k-\mu) \sigma}{(2 \omega+3)}-G_{0} A=1 \\
& +\frac{4 G_{0}(\mu-k)}{(2 \omega+3)} \ln (r / 2 a)-G_{0} A
\end{aligned}
$$

By choosing $A=\frac{4(\mu-k)}{(2 \omega+3)} \ln \left(r_{0} / 2 a\right)$, where $r_{0}$ is a constant, we get

$$
d s^{2}=\left[1+\frac{4 G_{0}(\mu-k)}{(2 \omega+3)} \ln \left(r / r_{0}\right)\right]
$$

$$
\left[\frac{d r^{2}}{\left(1-4 G_{0}(\mu-k)\right)}+r^{2} d \theta^{2}\right]
$$

Defining $d r^{\prime}=\frac{d r}{\sqrt{1-4 G_{0}(\mu-k)}}$, one can write the above equation as

$$
\begin{gathered}
d s^{2}=\left[1+\frac{4 G_{0}(\mu-k)}{(2 \omega+3)} \ln \left(r^{\prime} / r_{0}\right)\right] \\
{\left[d r^{\prime 2}+r^{\prime 2}\left(1-4 G_{0}(\mu-k)\right) d \theta^{2}\right]}
\end{gathered}
$$

Finally, if we put $k=-\mu$ for "string" matter we are led to

$$
\begin{gathered}
d s^{2}=\left[1+\frac{8 G_{0} \mu}{(2 \omega+3)} \ln \left(r^{\prime} / r_{0}\right)\right] \\
{\left[d r^{\prime 2}+\left(1-8 G_{0} \mu\right) r^{\prime 2} d \theta^{2}\right]}
\end{gathered}
$$

This is exactly the same metric for a straight vacuum string for section $t=$ constant and $z=$ constant which was earlier obtained by Barros and Romero[16] in BD theory in the weak field approximation. At this point, it is worthwhile mentioning that this spacetime has an angular deficit $\Delta \Phi$ given by

$$
\Delta \Phi=8 \pi G_{0} \mu\left[1-\frac{1}{2 \omega+3} \ln \left(\frac{r^{\prime}}{r_{0}}\right)\right]
$$

as may be seen directly by integrating (2.10) around a circle of radius $r^{\prime}$.

Finally, it is well known that in the weak field approximation when $\omega \rightarrow \infty$ the BD solution goes over to the corresponding solution in Einstein's GR, although this is not always true in the case of exact solutions [17]. In our case one can check that for $\omega \rightarrow \infty$, both $\epsilon_{1}$ and $\epsilon_{2}$ become zero and $G_{0} \rightarrow G$, so one can recover the corresponding GR solution for a circular cosmic string obtained by Hughes et al[6].

In conclusion, we have obtained the spacetime for circular cosmic string loop in BD theory in the weak field approximation. In doing so, we have followed the method prescribed by Barros and Romero[14]. When the loop is made of "string" matter we find out that at points near the string $(\sigma \rightarrow \infty)$ we recover the result previously obtained by Barros and Romero[16] for a straight static local string in BD theory. The solution goes over to the corresponding solution in GR in the limit $\omega \rightarrow \infty$. Hence We have generalised the result previuosly obtained by Hughes et.al [6] for circular cosmic string in GR, in Brans-Dicke theory.

As the trajectories of the light rays, which are given by the null geodesics, the only change involved in BD theory is the replacement of $G$ by an new $\omega$ dependent "effective" gravitational constant $G_{0}=\left(\frac{(2 \omega+3)}{(2 \omega+4)}\right) G$ and for $\omega$ to be consistent with solar system experiment and observation, $\omega \sim 500$ [18], this means that photons travelling in the spacetime will experience a decrease of gravitational constant as $G_{0} \sim 0.999 G$. Therefore, it 
follows that the distortion of the isotropy of the CMBR due to the gravitational field of the circular cosmic string loops in BD theory may be calculated directly from the results obtained in GR. A detail analysis of the full nonlinear BD field equations will certainly give more insight to the problem and for that a detail numerical calculation should be done which will be the aim of our future study.

\section{References}

[1] T. W. Kibble, J. Phys. A 9, 1387 (1976).

[2] A. Vilenkin, Phys. Rep. 121, 263 (1985).

[3] A. Vilenkin, Phys. Rev. D 24, 2082 (1981); J. R. Gott, Astrophys. J. 288, 422 (1985); T. Vachaspati and A. Vilenkin, Phys. Rev. D 31, 3052 (1985); R. Brandenberger, A. Albrecht and N. Turok, Nucl. Phys. B 277, 605 (1986).

[4] V. P. Frolov, W. Israel and W. G. Unruh, Phys. Rev. D 39, 1084 (1989)

[5] R. Arnowitt, S. Deser and C. W. Misner, in: Gravitation: An Introduction to Current Research, edited by L. Witten (Wiley, N. Y., 1962).

[6] S. J. Hughes, D. J. McManus and M. A. Vandyck, Phys. Rev. D 47, 468 (1993).
[7] D. J. McManus and M. A. Vandyck, Phys. Rev. D 47, 1491 (1993)

[8] M. B. Green, J. H. Schwartz and E. Witten, Superstring Theory (Cambridge University Press, Cambridge, England, 1987).

[9] C. Brans and R. H. Dicke, Phys. Rev. 124, 925 (1961).

[10] D. La and P. J. Steinhardt, Phys. Rev. Lett. 62, 376 (1989).

[11] A. H. Guth, Phys. Rev. D 23, 347 (1981).

[12] A. A. Sen, Phys. Rev. D 60, 067501 (1999).

[13] E. J. Copeland, E. W. Kolb and A. Liddle, Phys. Rev. D 42, 2911 (1990).

[14] A. Barros and C. Romero, Phys. Lett. A 245, 31 (1998).

[15] H. Bateman, Partial Differential Equations of Mathematical Physics (Cambridge University Press, Cambridge, England, 1952).

[16] A. Barros and C. Romero, J. Math. Phys. 36, 5800 (1995).

[17] C. Romero and A. Barros, Phys. Lett. A 173, 243 (1993); N. Banerjee and S. Sen, Phys. Rev. D 56, 1334 (1997).

[18] C.M.Will, Theory and Experiment in Gravitational Physics, Cambridge University Press (1993). 